\title{
Highly reliable Coordinated Control Strategy for Static Frequency Converters applied to Large Synchronous Condensers
}

\author{
Xiangjian Shi ${ }^{1,2}$, Teng $\mathrm{Liu}^{2}$, Wei Mu${ }^{2}$, Jianfeng Zhao ${ }^{1 *}$ \\ ${ }^{I}$ School of Electrical Engineering, Southeast University, Nanjing 210096, Jiangsu, China \\ ${ }^{2}$ NR Electric Co., Ltd., Nanjing 211106, Jiangsu, China \\ *Corresponding Author.
}

\begin{abstract}
A large synchronous condenser can quickly and flexibly implement various functions such as dynamic change in load tracking, realization of peak reduction and valley filling, emergency reserve, frequency modulation, and phase modulation. This type of condenser is typically started using a static frequency converter (SFC). Based on the analysis of the working principle of SFCs, this paper proposes a highly reliable coordinated control method with resynchronization criterion for an SFC. An auxiliary loop is introduced for voltage control at the SFC to realize feedforward of the trigger angle of the rectifier. This method can effectively improve the success rate of grid synchronization of the synchronous condenser and shorten the start-up time. Moreover, when the AC voltage on the grid side drops, the auxiliary ring can automatically reduce the terminal voltage to maintain a stable operation. A comparative experiment was conducted on an RTDS to verify the feasibility and effectiveness of the proposed method.
\end{abstract}

Keywords: static frequency converter, synchronous condenser, coordinated control, reentry synchronization, voltage dips

\section{Introduction}

Long-distance large-capacity transmission through ultra-high voltage direct current (UHVDC) transmission is an important means of solving China's new energy consumption problem and allocating optimal resources in the east and west. However, the stable operation of UHVDC converter station will be affected by the fluctuation of AC voltage. Therefore, UHVDC transmission converter stations require a high reactive power and fast support to ensure the stability of power system operation ${ }^{[1-3]}$. A large-capacity synchronous condenser can quickly and flexibly implement multiple functions such as dynamic change in load tracking, realization of peak reduction and valley filling, emergency reserve, frequency modulation, and phase modulation. It has been increasingly used in receive-side converter stations in UHVDC transmission.

Methods for staring up a large-capacity synchronous condenser include coaxial asynchronous motor, asynchronous start, and variable frequency start ${ }^{[4]}$. Currently, a synchronous condenser with a variable frequency is the main method that is being used ${ }^{[5]}$. The other methods cannot meet the large capacity requirement of synchronous condensers. The thyristor-based current source inverter, also known as the static frequency converter (SFC), can meet the startup requirements ${ }^{[8-11]}$. The condenser contains main excitation and auxiliary excitation. After the SFC drags the synchronous condenser, the commonly adopted grid-connection method is used for idling synchronization grid-connection: When the condenser starts, the auxiliary excitation is in operation mode, whereas the main excitation is not in operation mode. In addition, the rotor of the condenser is dragged to 1.05 times the rated speed in a short time through the variable frequency start system. The variable frequency start system is then stopped. After confirming the exit of the inverter startup system, auxiliary excitation is switched to main excitation ${ }^{[12-17]}$. The rated terminal voltage is established by controlling the excitation system. However, there is a probability that the grid connection may fail. Typically, a grid connection failure due to the unsatisfied conditions of synchronization can cause the system to enter fast restart. If the synchronization fails, a manual analysis needs to be performed to determine whether the fast restart conditions are met; this process takes a considerable amount of ISSN: 0010-8189

(C) CONVERTER 2020 
time. It is not conducive for the SFC to be operated quickly, as it reduces the availability of the SFC.

Dynamic behaviors and control methods of the start-up stage with SFC were compared and analyzed [16, 17], but it could not solve the problem of fast restart. The SFC logs out and the SC is connected to the power grid in the idling grid-connection stage. The constraint condition and success rate of grid-connection were studied [18]. In the reactive power regulation stage, the SC ensures the safe and stable operation of the power grid [19].

This paper first analyzes the working principle of an SFC applied to synchronous condensers. To shorten the time required for synchronous condenser grid connection, a highly reliable coordinated control method with resynchronization criterion for the SFC is proposed, and related controller parameters are designed. Finally, the control method is verified through the hardware in the loop simulation platform of RTDS, and the results are compared with those obtained using the conventional method.

\section{Configuration and Operation Principle of SFC}

An SFC uses a thyristor converter to convert the alternating current with power frequency into an alternating current with a continuously adjustable frequency. The variable frequency current is outputted to the stator winding of a synchronous motor to form a stator rotating magnetic field. Simultaneously, an exciting current is applied to the rotor to form a rotor magnetic field. The rotating stator magnetic field interacts with the rotor magnetic field to make the rotor to rotate; thus, the synchronous condenser can be started. The SFC mainly comprises a control system, two independent thyristor-based converters, and a DC circuit, as shown in Figure 1.

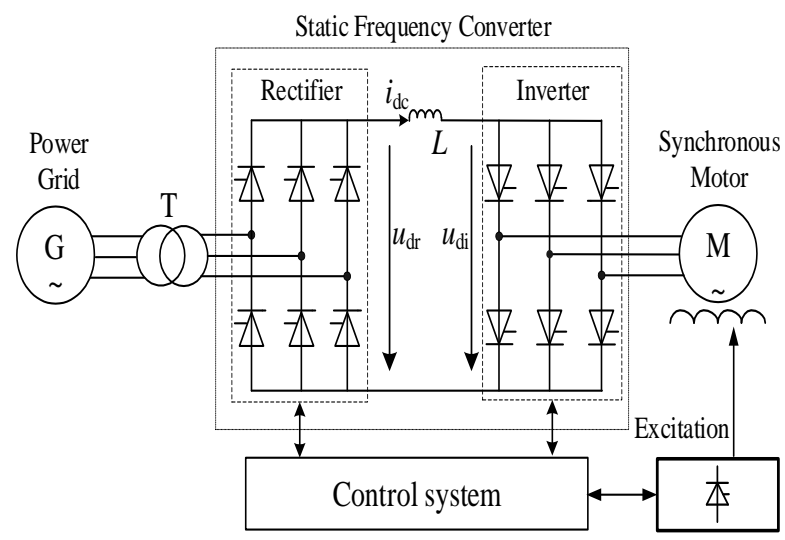

Fig. 1 Schematic circuit of SFC

The control system is responsible for the control and protection of the whole system, including rotor position detection, closed-loop speed control, trigger units of the thyristor, excitation system, and protection unit with overvoltage, overcurrent, di/dt, and flux protection. The two independent thyristor-based converters include a three-phase thyristor-based rectifier connected to the grid side as a power source and a three-phase thyristor-based inverter connected to the synchronous motor side. The rectifier and inverter are connected through the DC side. The rectifier or inverter can be a three-phase thyristor-based converter or in the form of a number of series thyristor-based converters. A smoothing direct-current reactor $L$ is set in the DC circuit to suppress the ripple of the DC current, limit the rise rate of the DC current, and decouple the two AC systems. In Figure 1, the input transformer $\mathrm{T}$ is a step-down transformer.

The start-up of the synchronous condenser realized by the SFC is a synchronous start up. Through a smoothing reactor, the output of the rectifier in the SFC forms a stable DC output. The inverter of the SFC converts the DC power into a frequency-variable AC power with a gradually increasing frequency based on the voltage frequency of the motor stator and outputs it to the windings of the motor stator. Thus, the stator rotating magnetic field that is ahead of the rotor magnetic field is generated. Through the interaction between the stator rotating magnetic field 
and the rotor magnetic field, an acceleration torque is generated, thus accelerating the motor rotor to the set speed. To achieve torque control, based on the rotor position or terminal voltage information of the motor, the SFC are controlled to gradually increase the alternating frequency for the feeding current of the two phases of the motor stator. It generates a continuous stator rotating magnetic field that is ahead of the rotor magnetic field. Through the interaction between the magnetic field and the rotor magnetic field, it controls the motor rotor to accelerate depending on the process and reach the setting speed. The electromagnetic torque is proportional to the magnitude of the DC current, the trigger angle of the inverter, and the magnitude of the magnetic flux of the condenser:

$$
T_{\mathrm{e}}=C I_{\mathrm{d}} \cos
$$

Here, $C$ is a coefficient, which is related to the characteristics of the unit; $T_{\mathrm{e}}$ is the electromagnetic torque, N. m; $I_{\mathrm{d}}$ is the DC current value of the static frequency inverter, $\mathrm{A} ; \cos (\varphi)$ is the power factor of the SFC; and $\Phi$ is the magnetic flux, $\mathrm{Wb}$.

When the trigger angle of the inverter is constant, the DC current $I_{\mathrm{d}}$ is adjusted via the closed-loop control; the magnetic flux $\Phi$ depends on the excitation control of the motor. During the startup process, the SFC controls the motor rotor excitation based on the drag torque. Taking the motor speed as the control target, we can control the acceleration torque by controlling $I_{\mathrm{d}}$ of the rectifier.

Because the three-phase stator current of the SFC is a trapezoidal wave in the working process, and only two phases are conducting at any time (except for the commutation phase), we used the conventional phasor analysis method and ignored the additional effects due to harmonic currents, such as torque ripple. Through the motor phasor diagram, some basic characteristics of the SFC during startup are explained.

Considering only the fundamental wave component, a phasor diagram of each electrical quantity of the motor can be drawn when the static inverter is dragging and running, as shown in Figure 2.

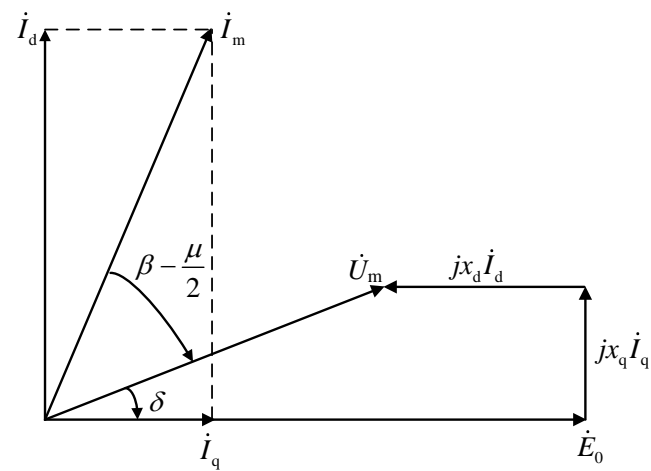

Fig. 2 Phasor diagram of motor operation

In the figure, $E_{0}$ is the motor no-load potential; $U_{\mathrm{m}}$ is the motor voltage; $I_{\mathrm{m}}$ is the motor stator current; $\delta$ is the internal power angle; $\mu$ is the inverter commutation overlap angle; $\beta$ is the commutation advance angle of inverter; $I_{\mathrm{d}}$ and $I_{\mathrm{q}}$ are the d-axis and q-axis components of the stator current, respectively. From the phasor diagram above, it can be seen that during the start-up process, the motor stator current $I_{\mathrm{m}}$ is ahead of the terminal voltage $U_{\mathrm{m}}$, and $U_{\mathrm{m}}$ is ahead of $E_{0}$. So the synchronous motor delivers reactive power and absorbs active power from the static frequency inverter.

\section{Coordinated Control Strategy for Static Frequency Converter}

\subsection{Implementation of Control Method}

ISSN: 0010-8189 


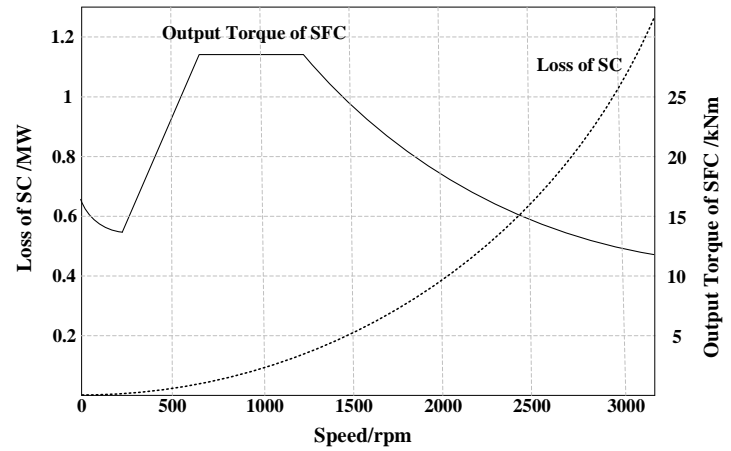

Fig. 3 The relationship curve of output torque of SFC and Loss of SC

In order to shorten the start-up time, the proposed high reliable coordinated control method includes two aspects. One is the resynchronization criteria, which can analyze the cause of the failure of the startup and upgrade from the time-consuming manual analysis to the program automatic judgment; the other is the bridge trigger feed forward technology, which can improve the output power of SFC and shorten the drag time of the condenser. The implementation of the coordinated control strategy can enhance the grid-connection success rate of the condenser. The grid connection failure due to unsatisfied synchronization conditions can cause the system to enter fast restart. Performing a manual analysis to determine whether the fast restart conditions are met after synchronization failure takes a long time, which is not conducive to the rapid commissioning of the unit, because it reduces the availability of the unit. Moreover, as shown in Fig. 3, in the high-speed stage, the unit loss is high, the output torque of the starting system is relatively low, and the unit rise rate is low.

Therefore, it is helpful to reduce the grid connection time by SFC and dragging as early as possible. To this end, we propose a reentry synchronization technology, where an intelligent monitoring system will automatically judge whether the synchronization instructions are issued, so as to determine whether the unit has the conditions for reentry into the synchronization. If the conditions are met, depending on the speed start function of the start system, the terminal voltage control technology is applied, the torque in the high-speed section is improved, and the unit automatically re-enters to start the grid connection process at the high-speed point. As shown in Fig. 4, four criteria must be met to issue the instructions for reentry synchronization: enable synchronous sequence control, operating frequency $f_{\mathrm{m}}$ is less than the minimum grid-connection frequency $f_{\min }$, grid-connection switch in open state, and no other alarms or failures. Through the reentry synchronization technology, the synchronous condenser can be rapidly reentry synchronized, thereby improving the success rate and availability of grid-connection.

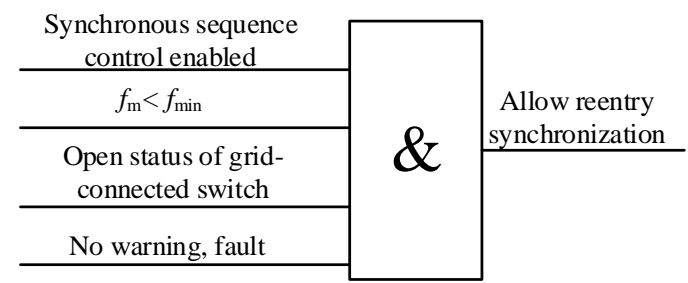

Fig. 4 Criteria for allowing reentry synchronization

To meet the requirements of the steadily rising motor speed, the SFC needs to provide a stable driving moment to the motor rotor. The moment depends on the variable frequency current of the SFC outputted to the motor stator and the magnitude of the excitation current. Under the condition of constant excitation current, the output current of the static inverter determines the driving torque, drag acceleration, and drag process. The SFC output current control is the means, and speed control is the target; therefore, a double closed-loop control is generally adopted for SFC control.

The outer loop of the double closed-loop control is the speed closed-loop, which is adjusted by PI (proportion and ISSN: 0010-8189 
integration), and the inner loop is the current closed-loop, which is also adjusted by PI. If the unit frequency can be measured, the deviation between the set and measured values of the unit speed can be calculated. After the calculation, PI control produces a given value of the rectifier output direct current. The difference between the given value of the DC current and the measured DC current is outputted by the rectifier. The control value calculated by PI adjustment is used to generate the trigger angle value by cosine phase shift, as shown in Fig. 5 .

Outer loop: The speed regulator aims to maintain the unit speed at the speed reference. The output value of the speed loop is used as the reference value of the inner loop-current regulator. The current regulator takes the control output current $I_{\mathrm{d}}$ equal to the current reference value $I_{\text {dref }}$ as the target, and the output value is used as the control value. The trigger angle $\alpha \mathrm{R}$ of the rectifier is obtained through calculation.

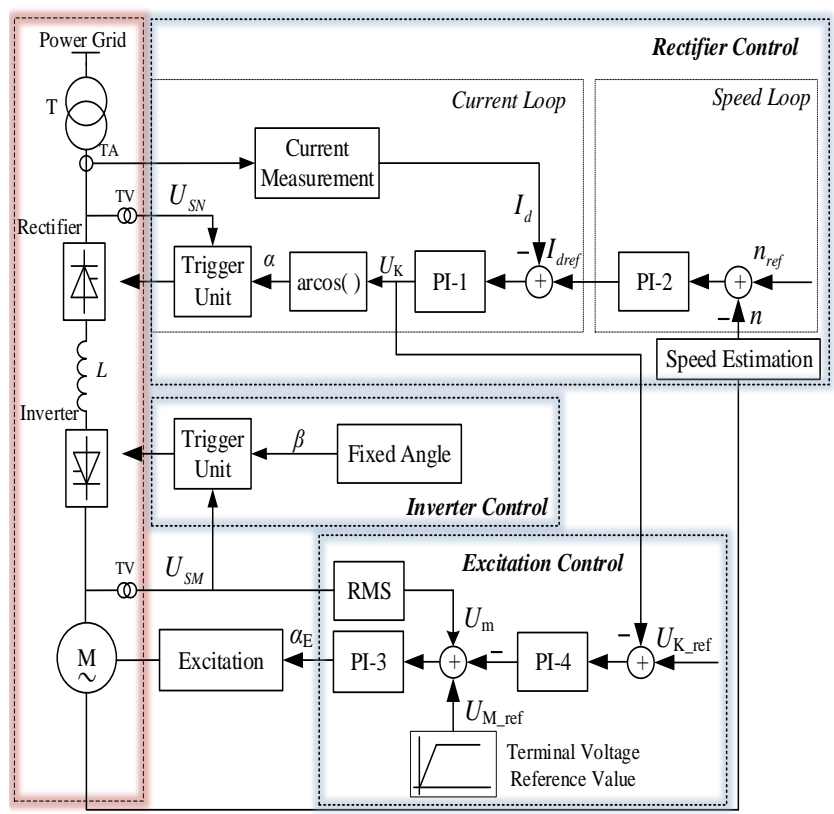

Fig. 5 Proposed coordinated control strategy for SFC

To give full play to the transformer capacity, the power factor of the rectifier should be improved, to enable the rectifier to work at small triggering angles. During the start-up process of the static inverter dragging the generator set, when the unit speed exceeds a certain value, the system will enter a field weakening zone. The static inverter system enters the power control mode. In the case of long-term continuous operation, under the premise of a certain system output power, in order to make full use of the primary voltage design margin of the static inverter, the reference voltage value at the machine end can be appropriately increased to increase the machine side direct voltage. This not only gives full play to the limit power of the system, but is also conducive to thyristor commutation, which is significant for the safe and reliable operation of the system.

However, the coordination of the operating points of the rectifier and inverter is the basis for a reliable power transmission in the SFC. If the machine-side direct voltage $U_{\mathrm{di}}$ is higher than the grid-side direct voltage $U_{\mathrm{dr}}$, the power transmission and safe operation of the system is affected. Therefore, while boosting the voltage at the machine end, a coordinated control of the inverter and rectifier of the SFC needs to be made to prevent the rectifier from limiting the trigger angle and causing the loop current to go out of control. To this end, when the SFC is running in the field weakening zone, the rectifier operating point (the rectifier trigger angle cosine value $U_{\mathrm{K}}=$ cos $(\alpha)$ as the representative quantity) is introduced as the direct control target. The closed-loop calculation output of its regulator PI-4 is as shown in Fig. 5. It is superimposed on the reference value $U_{\mathrm{M} \_ \text {ref }}$ of the generator voltage control loop, and a new reference value is obtained.

When the trigger angle cosine value $U_{\mathrm{K}}$ of the rectifier is lower than the reference value $U_{\mathrm{K}_{-} \text {ref }}$, under the action of 
the auxiliary loop PI-4, the excitation current is increased to increase the voltage at the synchronous condenser, and the DC voltage on the inverter side is slowly increased. To maintain the loop current, under the action of the automatic current regulator PI-1, $U_{\mathrm{K}}$ will increase and tend to the reference value.

Taking the operating point of the rectifier as the control target, we can appropriately adjust the terminal voltage, and the direct voltage margin of the system can be fully utilized to achieve maximum dynamic power output of the system and improve the input side power factor. When the system has maximum power output, the operating point of the rectifier is automatically adjusted. This increases the input power factor of the system, and the safe working area is not crossed.

\subsection{Parameter Design of Controller}

Assuming the motor is running in an initial state, the basic motion equation of the motor can be obtained as follows:

$$
\frac{G D^{2}}{375} \cdot \frac{d}{d t} n=\left(C_{M} I_{m}-C_{R} I_{m}^{2}\right)-M_{L}
$$

Here, $n$ is the motor speed, and $M_{\mathrm{L}}$ is the load mechanical torque, which is the resistance torque of the shaft system of the pumping unit during the start-up of the pumped storage unit.

According to Figure 1, the equation for the SFC can be obtained as follows:

$$
L \frac{d}{d t} I_{\mathrm{D}}+R_{\Sigma} I_{\mathrm{D}}=U_{d r}-C_{e} n
$$

Here, $C_{\mathrm{e}}$ is the motor counter electromotive force coefficient.

After standardizing the DC and motor currents, we have $I_{\mathrm{D}}=I_{\mathrm{m}}$. Therefore, $I_{\mathrm{D}}$ is used to characterize the DC and motor currents uniformly.

A signal analysis is performed on Equations (2) and (3), assuming that the average value of the rectifier direct voltage has a variable quantity $\Delta U_{\mathrm{dr}}$, and the applied load torque remains unchanged. Thus, we have:

$$
\begin{gathered}
L \cdot p\left(I_{\mathrm{D}}+\Delta I_{D}\right)+R_{\Sigma}\left(I_{\mathrm{D}}+\Delta I_{\mathrm{D}}\right)=U_{d r}+\Delta U_{d r}-C_{e}(n+\Delta n) \\
\frac{G D^{2}}{375} \cdot p(n+\Delta n)=\left[C_{M}\left(I_{\mathrm{D}}+\Delta I_{m}\right)-C_{R}\left(I_{\mathrm{D}}+\Delta I_{m}\right)^{2}\right]-M_{L}
\end{gathered}
$$

In (4), if we consider the electromagnetic transient process and ignore the motor speed change (assuming $\Delta n=0$ ), we can obtain the transfer function between $I_{\mathrm{D}}$ and $U_{\mathrm{dr}}$ :

$$
G_{2}(s)=\frac{I_{\mathrm{D}}(s)}{U_{d r}(s)}=\frac{1}{R_{\Sigma}\left(T_{L} s+1\right)}
$$

$T_{L}=\frac{L}{R_{\Sigma}}$ is called the electromagnetic time constant.

According to (5), ignoring the term $\Delta I_{\mathrm{D}}{ }^{2}$, we can obtain the transfer function between $I_{\mathrm{D}}$ and $n$ : 


$$
G_{\mathrm{M}}(s)=\frac{n(s)}{I_{\mathrm{D}}(s)}=\frac{R_{\Sigma}}{C_{e}} \frac{1}{T_{M}^{\prime} s}
$$

Here, $T_{M}^{\prime}=\frac{G D^{2}}{375} \frac{R_{\Sigma}}{C_{e}\left(C_{M}-2 C_{R}\right)}$ is called the electromechanical time constant.

Accordingly, an S-domain small signal block diagram of the SFC system can be obtained, as shown in Figure 6.

In the figure, $G_{1}=K_{1} /\left(T_{1} s+1\right)$ is the transfer function of the rectifier of the SFC, $K_{0}$ is the voltage magnification of the rectifier, and $T_{0}$ is the equivalent time constant, which is generally $0.167 \mathrm{~ms}$ for a six-pulse full-control bridge. $C_{\mathrm{n}}(s)=K_{\mathrm{p} 2}+K_{\mathrm{i} 2} / s$ and $C_{\mathrm{i}}(s)=K_{\mathrm{p} 1}+K_{\mathrm{i} 1} / s$ are the transfer functions of the speed PI regulator and the current PI regulator, respectively. To suppress the disturbance signal in the measurement, the speed and current feedback values are usually filtered. $H_{\mathrm{i}}(\mathrm{s})=K_{\mathrm{i}} /\left(T_{\mathrm{i}} s+1\right)$ and $H_{\mathrm{f}}(s)=K_{\mathrm{f}} /\left(T_{\mathrm{f}} s+1\right)$ are the equivalent transfer functions of the feedback measurement of the current and speed loops, respectively.

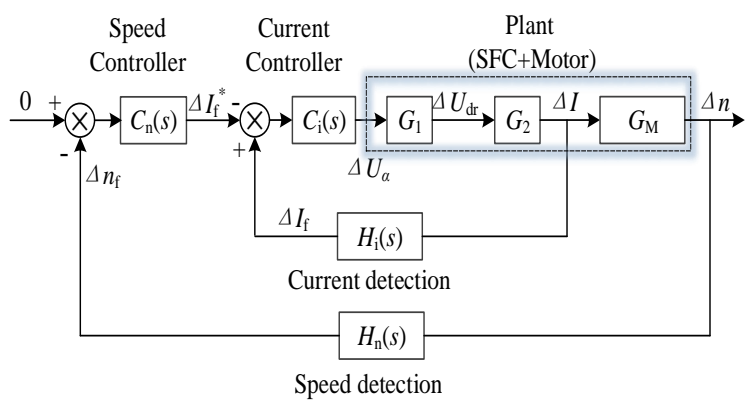

Fig. 6 S-domain small signal block diagram of the SFC system

In a double closed-loop control system, the structure and parameters of the speed and current regulators are selected depending on the actual requirement. With the help of the Bode plot design method, the open-loop frequency characteristics of the system are derived, and the expected characteristics of the system after correction are determined based on the performance indicators.

\section{Experimental Verification}

Table 1. Main parameters of experiment

\begin{tabular}{|c|c|}
\hline Parameter & Value \\
\hline Transformer turn ratio & $\begin{array}{c}10 \mathrm{kV} / 0.9 \mathrm{kV} / 0.9 \\
\mathrm{kV}\end{array}$ \\
\hline Direct-current reactor & $2.4 \mathrm{mH}$ \\
\hline Voltage of power grid & $500 \mathrm{kV}$ \\
\hline Rated power of Synchronous Condensers & $300 \mathrm{Mvar}$ \\
\hline Rated voltage of Synchronous Condensers & $20 \mathrm{kV}$ \\
\hline Rated current of Synchronous Condensers & $8660 \mathrm{~A}$ \\
\hline Rated speed of Synchronous Condensers & $3000 \mathrm{rpm}$ \\
\hline
\end{tabular}




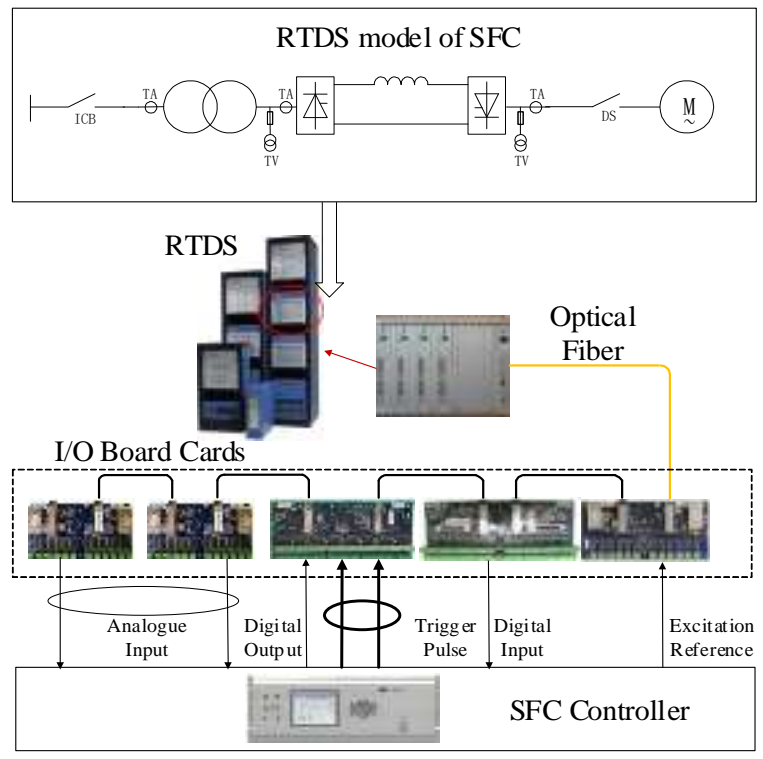

Fig. 7 RTDS platform of SFC

To verify the effectiveness and feasibility of the proposed method, experiments were set up on an RTDS semiphysical platform. Table 1 lists the specific parameters. As shown in Fig. 7, the platform includes an RTDS realtime digital emulator, an IO interface card, and the SFC controller. The IO card completes the mutual conversion of digital and analog signals; the RTDS real-time digital emulator and IO card are connected through an optical fiber, and the signal interface card is connected with the SFC control and protection equipment cable. The power supply, primary circuit, motor, and excitation system of the static inverter are realized in the form of a digital model in the RTDS simulator. The digital signals of the voltage, current, and breaker states of the primary part of the simulation are outputted to the SFC controller through the interface board. Based on these values, the controller takes the speed as the control object and outputs the trigger pulse. The control pulse is inputted to the RTDS model through the interface board to control the opening of the thyristor in the digital model and change the frequency and magnitude of the output current. After the stator of the motor model in the RTDS inputs the variable frequency current, the speed will increase, thus completing the simulation of the entire process.
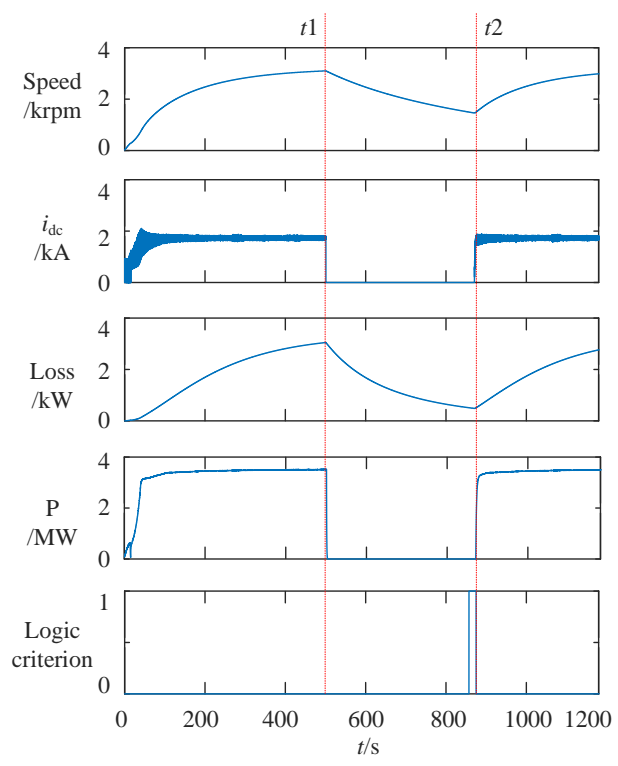

(a) Conventional method 

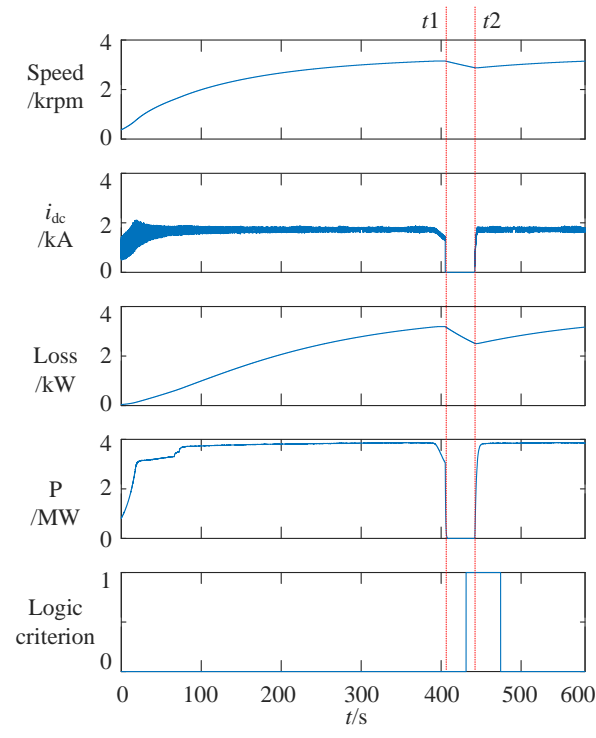

(b) Proposed method

Fig. 8 Resynchronization test waveform on RTDS

Figures 8 and 9 show the tests performed under two conditions: reentry synchronization and voltage drop.

Figure 8 shows the test waveforms during the synchronization process after grid connection failure. $t 1$ is the first synchronization time. After the synchronization failure, the resynchronization criterion is met at $t 2$. The system then enters the resynchronization process. A comparison of Figs. 8(a) and 8(b) shows that, through the selfdiagnosis criteria for resynchronization, the unit can realize a seamless connection between the two synchronizations. With the introduction of the grid bridge trigger angle feed forward control, the output power of the startup system is increased by approximately $15 \%$. The time required by the conventional method for the reentry synchronization process is $350 \mathrm{~s}$, and the time required by the proposed coordinated control method is $50 \mathrm{~s}$. The one-click startup realized with this method can improve the success rate of grid connection and significantly shorten the startup time.

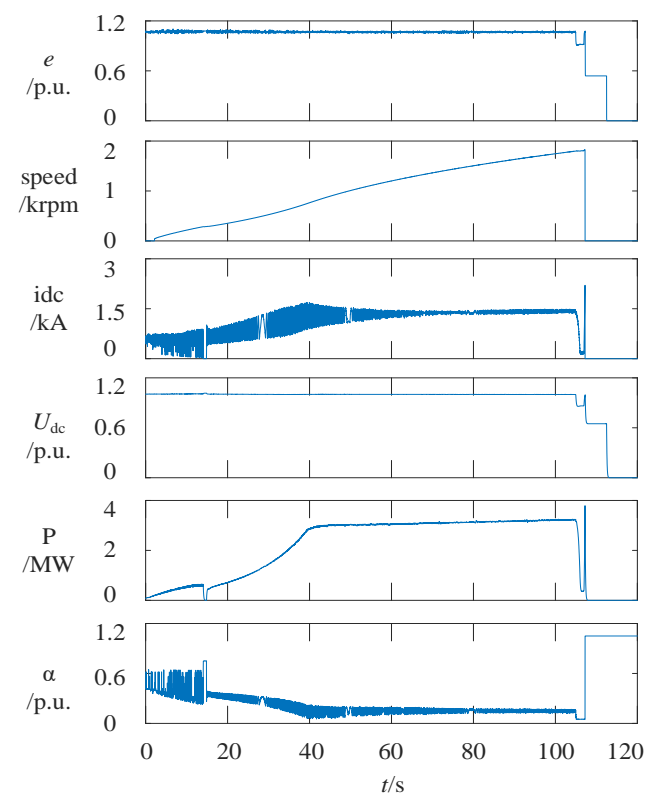

(a) Conventional method 


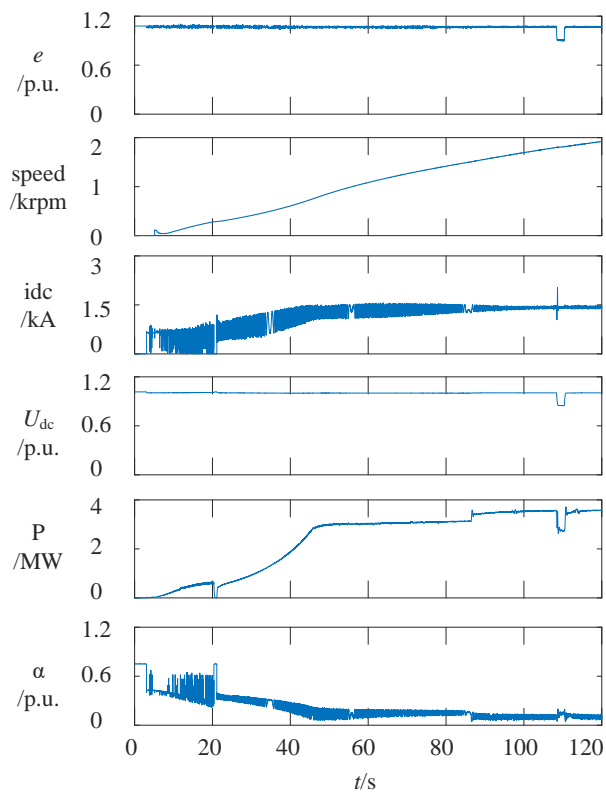

(b) Proposed method

Fig. 9 Voltage drop test wave on RTDS

Figure 9 shows the test waveform of the voltage drop of the power grid. At $100 \mathrm{~s}$, the power grid voltage drops $(15 \%, 2 \mathrm{~s})$, and the input current of SFC is over-current and triggers the protection trip under the conventional method. A stable start of the synchronous condenser cannot be achieved. Under the proposed coordinated control method, the auxiliary loop automatically reduces the terminal voltage, maintains the output power of the SFC system, and achieves a stable operation of the system.

\section{Conclusion}

In this study, the paper analyzed the working principle of an SFC system used for starting a synchronous condenser and developed a coordinated control method with resynchronization criterion for the SFC system to enhance the grid-connection success rate of the condenser and reduce startup time. In this method, an auxiliary loop for the voltage control at the SFC machine end is introduced to realize a feed forward control of the trigger angle of the grid bridge. The method was tested on an RTDS platform. The following conclusions can be drawn from the tests results:

(1) The method can realize a quick automatic restart of the unit and seamless connection during synchronization, reduce the condenser acceleration time, increase the availability of the condenser group, and increase the degree of automation of the entire station.

(2) When the AC voltage on the grid side drops, the auxiliary loop automatically reduces the terminal voltage and stabilizes the SFC output power.

(3) This method can give full play to the output capacity of the SFC, improve the output torque of the high-speed section, and improve the reliability of the system.

\section{References}

[1] X. Fan, Y. Zhou, L. Ruan, K. Zhou, T. Wang, et al., "Study on coordinated control strategy of reactive power compensation device in DC converter station with new-generation synchronous condensers," 2018 International Conference on Power System Technology, Guangzhou, pp. 2966-2971, 2018.

[2] K. Ramachandra Sekhar, N. Vinoth Kumar, Y. Harada, "Impact of renewable energy control center on 
voltage stability and transmission network efficiency in wind farm integrated grid," 2014 IEEE International Conference on Power Electronics, Drives and Energy Systems; Mumbai. pp. 1-6, 2014.

[3] X. Wang, X. Wei, Y. Meng, "Experiment on grid-connection process of wind turbines in fractional frequency wind power system," In: IEEE Transactions on Energy Conversion, vol. 30, no. 1, pp. 22-31, 2015, doi: 10.1109/TEC.2014.2358498.

[4] K. Givaki, H.M. Rahman, D. Vozikis, A. Giveki, "Analysis of integration of multi-terminal HVDC network to weak grids," In: The Journal of Engineering 2019, vol. 16, pp. 3219-3224, 2019, doi: 10.1049/joe.2018.8447.

[5] A. Rabiee, A. Soroudi, A. Keane, "Risk-Averse preventive voltage control of AC/DC power systems including wind power generation," In: IEEE Transactions on Sustainable Energy 2015, vol. 6, no. 4, pp. 1494-1505, 2015, doi: 10.1109/TSTE.2451511.

[6] Y. Pu, Q. Zhong, X. Li, Y. Hu, G. Xu, "Influence of synchronous condenser on voltage stability of HVDC," 2018 IEEE Power \& Energy Society General Meeting; Portland; pp. 1-5, 2018.

[7] Y. Zhuang, R.W. Menzies, O.B. Nayak, H.M. Turanli, "Dynamic performance of a STATCON at an HVDC inverter feeding a very weak AC system," In: IEEE Transactions on Power Delivery, vol. 11, no. 2, pp. 958-964, 1996, doi 10.1109/61.489357.

[8] S.S. Kalsi, D. Madura, M. Ingram, "Superconductor synchronous condenser for reactive power support in an electric grid," In: IEEE Transactions on Applied Superconductivity 2005, vol. 15, no. 2, pp. 21462149, 2005, doi: 10.1109/TASC. 849481.

[9] X. Fan, Y. Zhou, L. Ruan, K. Zhou, T. Wang, et al., "Study on transient reactive power characteristics of new-generation large synchronous condenser," 2018 China International Conference on Electricity Distribution; Tianjin, pp. 1851-1855, 2018.

[10] M. Wu, Z. Dong, P. Li, X. Ma, Z. Jin, et al., "Impact of new-type synchronous condenser on voltage stability of jarud sending-end system," 2018 International Conference on Power System Technology, Guangzhou; pp. 2654-2659, 2018.

[11] Z.H. Rather, Z. Chen, P. Thøgersen, P. Lund, "Dynamic Reactive Power Compensation of Large-Scale Wind Integrated Power System," In: IEEE Transactions on Power Systems, vol. 30, no. 5, pp. 25162526, 2015, Doi: 10.1109/TPWRS.2014.2365632.

[12] S. Teleke, T. Abdulahovic, T. Thiringer, J. Svensson, "Dynamic performance comparison of synchronous condenser and SVC," In: IEEE Transactions on Power Delivery, vol. 23, no. 3, pp. 16061612, 2008, Doi: 10.1109/TPWRD.2007.916109.

[13] Q. Wang, T. Li, X. Tang, F. Liu, J. Lei, "Study on the site selection for synchronous condenser responding to commutation failures of multi-infeed HVDC system," In: The Journal of Engineering, vol. 16, pp. 1413-1418, 2019, doi: 10.1049/joe.8807.

[14] H. Ryu, B. Kim, J. Lee, I. Lim, "A study of synchronous motor drive using static frequency converter," 2006 12th International Power Electronics and Motion Control Conference, Portoroz, pp. 1496-1499, 2006.

[15] X. Chai, C. Zhang, Y. Wang, J. Gao, H. Yang, "Analysis on idle-load grid-connection transient impact current of DFIG," 2011 International Conference on Electrical Machines and Systems, Beijing, pp. 1-5, 2011.

[16] P. Wang, Q. Mou, X. Liu, W. Gu, X. Chen, "Start-Up Control of a Synchronous Condenser Integrated HVDC System with Power Electronics Based Static Frequency Converter," In: Access, vol. 7, no. 1, pp. 146914- 146921, 2019, doi: 10.1109/IFEEC.7992178.

[17] P. Wang, X. Liu, Q. Mou, W. Gu, X. Zhao, "Start-Up Control and Grid Integration Characteristics of 300 MVar Synchronous Condenser with Voltage Sourced Converter-Based SFC," In: Access, vol. 7, no. 1, pp. 176921- 176934, 2019, doi: 10.1109/ACCESS.2953052.

[18] H. Yang, Y. Zheng, X. Wang, Y. Jian, J. Hu, "Calculation Method of Success Rate for Idling Gridconnection of Large Synchronous Condensers," In: Automation of Electric Power Systems, vol. 42, no. 24, pp. 74-78, 2018, doi: 10.7500/AEPS20180320003.

[19] H. Zhang, J. Hasler, N. Johansson, L. Ängquist, H. Nee, "Frequency response improvement with synchronous condenser and power electronics converters," 2017 IEEE 3rd International Future Energy 
CONVERTER MAGAZINE

Volume 2021, No. 4

Electronics Conference and ECCE Asia, Kaohsiung; pp. 1002-1007, 2017. 